\title{
Foundations of fundamental mechanics
}

\author{
I \\ Thomas Blommaert \\ and \\ Michael Appleby \\ July 19, 2019 \\ The Netherlands
}

\begin{abstract}
The following paper presents a description on the fundamental mechanics of nature.

This is the first of a set of papers entitled Foundations of fundamental mechanics, in which this first paper is specifically on the nature of gravity.

For all intents and purposes this paper is NOT intended to be a replacement for the General theory of Relativity (GR) (A. Einstein 1915-1916), rather it is intended to be a

complimentary extension of its work, with the purpose of extending it into quantum physics. Most notably, to relate it to quantum field theory (QFT), by quantizing the metric of spacetime into a potential field theory.
\end{abstract}

Keywords: General Relativity, Quantum Mechanics, Cosmology, Quantum gravity. 
- Foundations of fundamental mechanics -

Whatever way it comes out, nature is there, and she is going to come out the way she is. Therefore, when we go to investigate it, we should not pre-decide what it is we are trying to do, except to find out more about it!*

- Richard Feynman.

*The Pleasure of Finding Things Out - BBC Horizon, 1981. 


\section{§ 1. Introduction}

When contemplating on the most fundamental levels of nature (e.g. highest energies, shortest lengths and smallest fractions of time), in regards to scales currently beyond our means of direct measurement or observation, the closest indications we have on such scales, are based on a set of natural units known as the Planck units (proposed by M. Planck, 1899).

The goal of the investigation laid out in this paper, is to obtain new insights on the nature of gravity, for the purpose of expanding (or rather, contracting...) it onto the quantum scale.

By means of dimensional analysis, mechanical interpretation, and use of some of the corresponding equations in a less conventional way (without the need for mathematical complexity), it is shown that additional information has been able to be found that may lead to a better understanding of gravitation on the quantum level of nature.

- In this paper we make use of the SI system of units. 


\section{§. Space-time and matter}

When attempting to derive the Planck energy $\left(E_{\mathrm{p}}\right)$ in terms of the Planck-Einstein relation i.e.:

$$
E=h v=\frac{h c}{\lambda}
$$

It shows that where $v=v_{\mathrm{p}}$ (where $v_{\mathrm{p}}$ is assumed to be the Planck frequency), we find that $v_{\mathrm{p}} \neq \frac{c}{l_{p}}$, where $l_{p}$ (the Planck length) represents the 'minimum' scale for the wavelength $(\lambda)$.

Instead it is found that:

$v_{\mathrm{p}}=\frac{c}{l_{p} 2 \pi}$, where $l_{p} 2 \pi=\lambda_{\mathrm{p}}$ and $l_{p}=\sqrt{\frac{\hbar G}{c^{3}}},\left(\hbar=\frac{h}{2 \pi}\right)$

consequently:

$\nu_{\mathrm{p}}=\sqrt{\frac{c^{5}}{\hbar G 2 \pi^{2}}}$ and $\nu_{\mathrm{p}} 2 \pi=\omega_{\mathrm{p}}=\sqrt{\frac{c^{5}}{\hbar G}}$, where $\omega_{\mathrm{p}}$ is the Planck 'angular' frequency.

Therefore:

$$
E_{\mathrm{p}}=\hbar \omega_{\mathrm{p}}=\frac{h c}{\lambda_{p}}
$$

From this we are able to derive the Planck mass $\left(m_{\mathrm{p}}\right)$, through the mass-energy equivalence:

$$
m_{\mathrm{p}}=\frac{E_{p}}{c^{2}}
$$

Where $m_{\mathrm{p}}=\sqrt{\frac{\hbar c}{G}}$, and $E_{\mathrm{p}}=\sqrt{\frac{\hbar c^{5}}{G}}$

By relating the Planck mass to the Newtonian law of gravitation 'for a single body' (i.e. $g=-\frac{G M}{r^{2}}$, where $r=l_{p}$, and $M=m_{\mathrm{p}}$ ), with respect to the speed of light $c$ in vacuum, it is found that:

$$
\frac{-\frac{G M}{r^{2}}}{c}=-\frac{G M}{r^{2} c}=\omega_{\mathrm{p}}
$$

Where $E_{\mathrm{p}}=\hbar \omega_{\mathrm{p}}$

Here we find that the former relation can be applied to further fundamental masses, (such as the electron etc.), as long as $r=l_{p}$ remains unchanged.

E.g. $-\frac{G M_{e}}{r^{2} c}=\omega$, where $E_{e}=\hbar \omega$ 
As the relation $E=h v$ relates the energy of a photon to its frequency, the former can be taken to indicate an equal relationship for a massless object who's energy is equivalent to that of the mass particle.

If we consider an object of energy equal to the Planck energy $\left(E_{\mathrm{p}}\right)$, we find that where $E_{\mathrm{p}}=\frac{h c}{\lambda_{p}}$, then relative to this relationship $\lambda_{\mathrm{p}} \neq r_{\mathrm{s}}$, (where $r_{\mathrm{s}}=\frac{2 G M}{c^{2}}$, the Schwarzschild radius, ${ }^{*}$ and $M=m_{\mathrm{p}}$ ), instead it is found that $\lambda_{\mathrm{p}}>r_{\mathrm{s}}$.

From this it can be inferred that as $-\frac{G M}{r^{2} c}=\omega$, and $E=\hbar \omega$, that $E$ indicates the energy of an occurrence related to the gravitational action of a particle of mass, and its energy.

$$
-\frac{G M}{r^{2} c}=\omega \rightarrow E=\hbar \omega=\mathrm{G}_{g}
$$

(Here subscript $g$ is used to distinguish from $\mathrm{G}$ as used for the Newtonian gravitational constant.)

Furthermore, it is found that as $\frac{c}{\omega_{p}}=l_{p}$, and $\frac{l_{p}}{c}=t_{p}$, (where $t_{p}=\sqrt{\frac{\hbar G}{c^{5}}}$ ' 'the Planck time'.

And $\frac{c}{v_{p}}=\lambda_{\mathrm{p}}$, where $\frac{\lambda_{p}}{c}=t^{\prime}$, then:

$$
t^{\prime}-t_{p}=\Delta_{\vec{t}}
$$

Where the direction of a change in time $\Delta_{\vec{t}}=t_{p} \rightarrow t$ relative to all physical events arising from the quantum scale.

Also, as $l_{p}=\sqrt{\frac{\hbar G}{c^{3}}} \rightarrow l_{p}^{2}=\frac{\hbar G}{c^{3}}=\frac{h G}{2 \pi c^{3}}=\frac{1}{2 \pi} \frac{h G}{c^{3}}$ where $\left(\frac{1}{2 \pi}=\frac{8 \pi}{4 \pi^{2}}\right)$

When rearranging:

$l_{p}^{2}=\frac{8 \pi G}{c^{3}} \frac{h}{4 \pi^{2}}=\frac{8 \pi G}{c^{4}} \frac{h c}{4 \pi^{2}}$

Where the latter shows the constant of the Einstein field equation (GR).

*The radius of a boundary within which a given mass would become a 'non-rotating' black hole (derived by K. Schwarzschild, 1916). 
As

$l_{p}^{2}=\frac{\hbar G}{c^{3}}$, then $G=\frac{l_{p}^{2}}{\frac{\hbar}{c^{3}}}$

Which can be written as:

$\frac{1}{c^{2}} \frac{\hbar G}{c} \rightarrow G=\frac{l_{p}^{2}}{\frac{1}{c^{2}} \frac{\hbar}{c}}$ where $\frac{\hbar}{c}$ in turn can represent $m \lambda,\left(\lambda=\frac{\lambda}{2 \pi}\right)$

If $m$ indicates the mass equivalence of a particle wave ( $§ 3$.$) , and \chi$ is the 'angular' wavelength of that particle wave, we find that:

$G=\frac{l_{p}^{2}}{\frac{1}{c^{2}} m \lambda}=\frac{l_{p}^{2}}{\frac{m \lambda}{c^{2}}}$

\section{$\S$ 3. Particle wave}

We propose the existence of a 'particle wave' (denoted as $\psi$ ), which is generated by the interaction of a mass particle with the (quantum) vacuum of the surrounding space-time.

Here we define what is meant by the particle wave:

The concept holds a close resemblance to that of the de Broglie matter wave hypothesis, ${ }^{*}$ and can - in some sense - be considered analogous to the concept of gravitational wave(s), ${ }^{* *}$ on a quantum scale.

At its basics it follows the same relationship as that of the matter wave, in terms of its wavelength and the momentum of the particle inducing the particle wave.

Further, as a vacuum state is known not to be empty, but instead constantly fluctuates with "virtual particles", ${ }^{[4][5][6]}$ and (in accordance with the indeterminacy principle - W. Heisenberg, 1927), ${ }^{[3]}$ as fundamental particles in their lowest energy state (even at zero temperature), are known to maintain some energy (through interaction with the quantum vacuum), which in turn is expected to lead to a form of "trembling motion" (zitterbewegung) ${ }^{[2]}$ (proposed by E. Schrödinger, 1930).

Then assuming that the particle wave is produced by the interaction of a (mass) particle with the quantum vacuum of surrounding space-time, it can be inferred that the particle wave itself is massless, and its velocity must therefore be equal to the speed of light $c$ in vacuum.

This makes it conceptually and fundamentally different from the matter wave concept.

- As well as it is a 'physical' occurrence and not only a mathematical concept.

\footnotetext{
* The concept that, as light exhibits wave and particle-like properties, so does matter (particles) have wave-like properties, (proposed by L. de Broglie, 1924).

**Gravitational waves have been predicted by the general theory of relativity. General relativity states that mass (matter) distorts space-time, when an object accelerates (e.g. orbital), it creates ripples in spacetime, emitting gravitational waves that carry energy away from the source. ${ }^{[1]}$
} 
The de Broglie wavelength for a matter wave is derived as:

$\lambda=\frac{h}{p}=\frac{h}{m \mathrm{v}}$ where $p=\frac{h}{\lambda}$ (for a mass particle, where $\mathrm{v}<$ or $\ll c$ )

For a massless particle, where $m=0$ (zero rest mass) and $\mathrm{v}=c$ :

$p=\frac{E}{c}=\frac{h}{\lambda}$ where $p \neq m \mathrm{v}$

The matter wavelength $\lambda=\frac{h}{p}=\frac{h}{m v}$ (for a mass particle), compared to $\lambda$ for a massless particle, where $\lambda=\frac{h}{p}=c t$

(as: $h=E \cdot t,\left(\right.$ Joule-second), and $\left.p=\frac{h}{\lambda}=m \mathrm{v}\right)$

Then, for a particle wave (massless):

$p=\frac{h}{\lambda}=m \mathrm{v}$, has to equal $p=\frac{h}{\lambda}=\frac{E t}{c t}=\frac{E}{c}$

Where

$p=\frac{E}{c}=m \mathrm{v} \rightarrow E_{\psi}=m \mathrm{v} \cdot c=p c\left(\right.$ and $\left.p_{\psi}=\frac{E_{\psi}}{c}\right)$.

Then

$E_{\psi}=m \mathrm{v} \cdot c \neq m c^{2}$

This implies that:

As $m=\frac{E}{c^{2}}$, then $m_{\psi}=\frac{E_{\psi}}{c^{2}}=\frac{p_{\psi}}{c}=\frac{m \mathrm{v}}{c}$

When the particle relating to the particle wave is at 'rest' (in the absence of linear velocity), we find:

$m_{\psi} c=m_{0}$

Therefore, as $p_{\psi}=\frac{E_{\psi}}{c}=m_{\psi} c=m \mathrm{v}$, then when at rest:

$p_{\psi}=m_{\psi c} \rightarrow \lambda=\frac{h}{p} \neq 0$ 
Here

$\lambda=\frac{h}{m_{\psi} c}$

From the former, it can be taken that relative to momentum:

$p_{\psi}=p \quad\left(\right.$ where $\left.m_{\psi c} c=m \mathrm{v}\right)$

In relation to kinetic energy $(\mathrm{KE})$ however, we find:

Where $\mathrm{KE}=\frac{1}{2} m \mathrm{v}^{2}$ for a matter particle, $\mathrm{KE}_{\psi}$ for the particle wave can be calculated from $\frac{p_{\psi}{ }^{2}}{2 m_{\psi}}$

This shows that, even though $p_{\psi}=p, \mathrm{KE}_{\psi}>\mathrm{KE}$

\section{§ 4. Conclusions}

By dimensional analysis (e.g. of Planck units), rearrangement of formulations and combining results with further corresponding equations, additional information has been found relating to the nature of gravity.

Through this work, it has become apparent that fundamental particles of mass interact with space-time by means of their 'particle wave'.

The concept of the particle wave has been proposed, and in turn, it has been found that quantization of space-time, relative to the interaction with fundamental particles, leads to the phenomenon of gravitation.

As this interaction occurs on the most fundamental level(s) of nature, where all 'physical' actions arise from the quantum scale, it has also shown to indicate that (on these scales) this leads to an apparent direction for the aspect of time in relation to the events.

\section{Suggestions for possible experiments:}

If a setup is arranged whereby a double set of uncharged conductive plates are placed in a vacuum (i.e. Casimir effect - H. Casimir, 1948), ${ }^{[7]}$ at adequate distance, such that there is no disturbance in the outcome of the measured force(s) between each set, at the closest possible range between the sets. Then when electrons are past between them (linearly), this should produce a detectable interference in the outcome of the measurement, due to the particle wave(s) (from the passing electron(s)) acting on the plates.

This can be taken as an indication of the physicality of the particle wave(s), as the electrons themselves do not directly interact with the plates. 
- Foundations of fundamental mechanics -

\section{References}

[1] Davide Castelvecchi, Alexandra Witze. Einstein's gravitational waves found at last. doi: 10.1038/nature.2016.19361

[..] Abbott BP, et al. (LIGO Scientific Collaboration and Virgo Collaboration). Observation of Gravitational Waves from a Binary Black Hole Merger. Physical Review Letters. 116, 061102 (2016)

[2] L.J. Leblanc, M.C. Beeler, K. Jimenez-Garcia, A.R. Perry, S. Sugawa, R.A. Williams and I.B. Spielman. Direct observation of zitterbewegung in a Bose-Einstein condensate. New Journal of Physics 15, 073011 (2013)

[3] D. Sen. The uncertainty relations in quantum mechanics. Current Science. 107, NO. 2, 203-218 (2014)

[..] P. Busch, P. Lahti, R. F. Werner. Proof of Heisenberg's Error-Disturbance Relation. Physical Review Letters. 111, 160405 (2013)

[4] C. Riek, D. V. Seletskiy, A. S. Moskalenko, J. F. Schmidt, P. Krauspe, S. Eckart, S. Eggert, G. Burkard, A. Leitenstorfer. Direct sampling of electric-field vacuum fluctuations. Science. 350, Issue 6259, 420-423 (2015). doi: 10.1126/science.aac9788

[5] A. Cho. Astronomers spot signs of weird quantum distortion in space. Science. (2016) doi: $10.1126 /$ science.aal0437

[..] R. P. Mignani, V. Testa, D. González Caniulef, R. Taverna, R. Turolla, S. Zane, K. Wu. Evidence for vacuum birefringence from the first optical-polarimetry measurement of the isolated neutron star RX J1856.5-3754. Monthly Notices of the Royal Astronomical Society. 465, Issue 1, 492-500 (2017). doi: 10.1093/mnras/stw2798

[6] Jérôme Martin. Everything you always wanted to know about the cosmological constant problem (but were afraid to ask). Comptes Rendus Physique 13.6-7, 566-665 (2012). (pp. 61-69)*

[7] W. M. R. Simpson, U. Leonhardt. Forces of the Quantum Vacuum: An introduction to Casimir physics. World Scientific. (2015). ISBN: 978-981-4632-90-4 